\title{
ACTITUD EMPRENDEDORA Y CAPACIDAD DE CREACIÓN DE MICROEMPRESAS EN ESTUDIANTES UNIVERSITARIOS DE SAN MARTÍN, PERÚ
}

\section{(Entrepreneurial attitude and capacity to create micro- enterprises in university students from San Martín, Peru)}

\author{
Robin Díaz Saavedra \\ robin.diaz@upeu.edu.pe \\ https://orcid.org/0000-0003-2707-8193 \\ Universidad Peruana Unión, Perú \\ Karel Meléndez Ruíz \\ fiorella.melendez@upeu.edu.pe \\ https://orcid.org/0000-0002-3667-4053 \\ Universidad Peruana Unión, Perú
}

\author{
Jheisy Rafael Chauca \\ jheisyrafael@upeu.edu.pe \\ https://orcid.org/0000-0003-3035-0899 \\ Universidad Peruana Unión, Perú \\ Luis Geraldo Campos \\ luis.geraldo@upeu.edu.pe \\ https://orcid.org/0000-0002-8366-689X \\ Universidad Peruana Unión, Perú
}

\begin{abstract}
RESUMEN. El emprendimiento puede empezar de manera empírica o desde las aulas. Este último debe ser una prioridad para las universidades públicas y privadas, por lo tanto, se debe empezar conociendo esa actitud y capacidad que tiene el estudiante para crear su propio negocio. En tal sentido la presente investigación plantea como objetivo principal determinar la relación que existe entre la actitud emprendedora y la capacidad de creación de microempresas en estudiantes de la carrera de Administración de tres universidades en la Región de San Martin, Perú. La investigación está basada en una metodología cuantitativa, no experimental, transversal, con alcance descriptivo y correlacional; para ello se adaptó y validado un instrumento mediante jueces que agrupa el constructo actitud emprendedora y la capacidad de creación de microempresas con valores finales de escala Likert que se aplicó a una muestra conformada por 131 estudiantes del VIII y IX ciclo de la carrera de Administración. Los resultados mostraron que un 65,6\% de los estudiantes mostraron que a menudo tienen una actitud emprendedora y el 60,3\% a menudo señalan que tiene la capacidad de crear microempresas; además, se encontró que la actitud emprendedora y la capacidad de creación de microempresas tiene una relación positiva y significativa $\left(R h o=0.656^{* *} ; p=0.000\right)$. En conclusión, se determina que mientras mayor sea la actitud emprendedora mayor será su capacidad de creación de microempresa.
\end{abstract}

Palabras clave: actitud emprendedora, conocimiento empresarial, creación de microempresa, emprendimiento, motivación empresarial.

\begin{abstract}
Entrepreneurship can start empirically or from the classroom. The latter should be a priority for public and private universities, therefore, you should start by knowing that attitude and ability that the student has to create their own business. In this sense, the main objective of this research is to determine the relationship between the entrepreneurial attitude and the ability to create micro-businesses in Administration students from three universities in the San Martin Region, Peru. The research is based on a quantitative, non-experimental, cross-sectional methodology, with a descriptive and correlational scope; For this, an instrument was adapted and validated by judges that groups the entrepreneurial attitude construct and the capacity to create micro-businesses with final Likert scale values that were applied to a sample made up of 131 students from the VIII and IX cycles of the Administration career. The results showed that $65.6 \%$ of the students showed that they often have an entrepreneurial attitude and $60.3 \%$ often indicate that they have the ability to create micro-businesses; Furthermore, it was found that the entrepreneurial attitude and the ability to create micro-businesses have a positive and significant relationship $\left(\mathrm{Rho}=0.656^{* *} ; \mathrm{p}=0.000\right)$. In conclusion, it is determined that the greater the entrepreneurial attitude, the greater will be their ability to create a micro-business.
\end{abstract}

Keywords: entrepreneurial attitude, business knowledge, micro-business creation, entrepreneurship, business motivation. 


\section{Introducción.}

En la actualidad existe una gran preocupación por mejorar la economía y la calidad de vida de la sociedad, y la actividad empresarial de la mano con las microempresas y las pequeñas empresas son las impulsoras fundamentales en el logro de una economía globalizada (AlMamun, et al., 2016, 2019; Nabiswa y Mukwa, 2017; Zea-Fernández, et al., 2020).

Las empresas son las que contribuyen a cerrar la brecha del desempleo, sin embargo, los nuevos emprendimiento que surgen de la informalidad o de iniciativas de estudiantes universitarios se debe brindar mayor apoyo, por lo que es bien sabido que un individuo con actitud emprendedora no copia iniciativas de emprendimiento, sino que se esfuerza por desarrollar y crear cosas nuevas o innovar sobre lo que ya existe con una alta persistencia, hasta logarlo (Contreras y Macías, 2021; Durán-Aponte y Arias-Gómez, 2016), en cambio dependerá de su capacidad de creación de empresas. Se entiende por capacidad de creación de empresas, al proceso de creación de la misma y que surgen en la combinación de la oportunidad de negocio, el equipo emprendedor y los recursos (Martínez, Durán y Serna, 2021; Radovich, 2017).

Si se habla del problema de emprendimientos a nivel internacional, en Estados Unidos la incertidumbre de emprender asciende al 27\%, cambio España representa casi el 50\%, haciendo de ello el país con más complejidad en cuestión de emprender (Olozagaste, et al., 2017). En tal sentido, Ortiz (2016) afirma que los estudiantes presentan una baja actitud emprendedora, debido a que cuando egresan de sus centros de estudios optan por ser funcionarios y competir por un puesto de trabajo, y no se sienten en la capacidad de emprender un negocio.

En el marco del desempleo, el Perú a través del Instituto Nacional de Estadística e Informática (INEI) reportó que en el periodo 2007 al 2017 mostró una tasa de desempleo juvenil de crecimiento negativo del $-0.6 \%$, y los jóvenes de 14 a 29 años con desempleo ascendieron a 450400 , siendo los hombres quienes tienen mayor representatividad en este índice (54.2\%) (INEI, 2018). Estos indicadores 
no son alentadores para el país, puesto que puede generar pobreza al no cubrir las necesidades diarias. Por lo tanto, es necesario establecer estrategias que ayuden a reducir la brecha del desempleo, una de estas seria mediante el sistema de educación, donde fomente a profesionales emprendedores que sean capaces de formar y crear empresas, de los cuales puedan plantear metas ambiciosas y ser competitivos en el ámbito laboral y académico, a fin de contar con más jóvenes emprendedores.

En este contexto, Serida, et al. (2018) elaboraron un informe en el cual mencionan que el $43 \%$ de los peruanos están preparados para iniciar un negocio en los próximos tres años. Este porcentaje permitió al Perú posicionarse en el puesto seis en cuanto al propósito de emprender en la región, a diferencia de Colombia quien lidera el ranking Latinoamericano sobre emprendimiento con 53\%, seguido de Ecuador $\operatorname{con} 48 \%$.

Datos revelan que el Perú cuenta con una tasa de mortandad de emprendimientos del $6.2 \%$ frente a $5.2 \%$, y con un nivel bajo de innovación del $17.8 \%$ frente a $23 \%$ del promedio en la región, ello hace necesario fortalecer la actitud emprendedora de la mayoría de la población, no bastando con un grupo menor, por lo que, se debe explotar al máximo su potencial a fin de generar soluciones innovadoras (El Comercio, 2018; Gestión, 2018). Empero, los jóvenes en la actualidad requieren mayor motivación y competencia dentro de los métodos educativos, toda vez que la incorporación del mercado laboral avanza en grandes rasgos, por lo que se debe apoyar a las iniciativas de emprendimiento surgidas en la academia, brindando asesoramiento y financiamiento para llevarlas a cabo, con fines de generar empleo en el país.

El Texto único ordenado de la Ley de impulso al desarrollo productivo y al crecimiento empresarial (2013) señala que el estado peruano considera como parte de su política la formalización, progreso y competitividad de las MYPEs y la base sostenible para los recientes emprendimientos, mediante el Gobierno Nacional, Regional y Local; ello, constituye un marco legal y promueve la inversión privada, forjando una oferta de servicios empresariales propuestos a optimizar los niveles 
de organización, administración, tecnificación y articulación productiva y comercial con las MYPE, así tendremos políticas que fortalezcan la organización, asociación empresarial y el incremento económico con empleo sostenible. Cabe precisar que, en el Perú, el índice de quiebra de las empresas es muy elevado. En el cuarto trimestre del año 2018 se crearon 70547 empresas, pero se dieron de baja 45 052, este resultado significó una variación neta de 25495 unidades económicas (INEI, 2019).

Según Muñóz y Martínez (2020) así como Radovich (2017), la actitud emprendedora y la capacidad de creación de microempresas tienen una relación significativa. Sin embargo, se desconoce si el objetivo de las universidades consiste en formar profesionales emprendedores, es decir, capacitados para crear su propio negocio, el cual requiere responsabilidad social y el cuidado de medio ambiente, pues, no debería existir planes de estudio en las escuelas profesionales de universidades que formen profesionales que se limiten a competir por puestos de trabajo ofertados en el mercado laboral, sino que, deben formar profesionales con aptitud de liderar y crear microempresas, sin depender de terceros.

Bajo esta perspectiva, el presente estudio pretende estudiar la actitud emprendedora y la capacidad de creación de microempresas en los centros de formación profesionales de la Universidad Científica del Perú (UCP), la Universidad Alas Peruanas (UAP) y la Universidad Nacional de San Martin (UNSM), siendo estos centros los más distinguidos y reconocidos dentro de la región de San Martin, Perú. Por lo tanto, el objetivo del presente estudio es determinar cuál es la relación entre la actitud emprendedora y la capacidad de creación de microempresas en los estudiantes de Administración de la UCP, UAP y UNSM.

\section{Metodología.}

El presente estudio utiliza un enfoque cuantitativo de tipo aplicada, con el propósito determinar mediante el conocimiento científico los medios que servirán para atender una necesidad 
identificada y específica (Ato, et al., 2013; Hernández, et al., 2014). Este tipo de enfoque está orientado a conocer y perseguir la resolución de problemas. Además, se caracteriza por ser un estudio de alcance descriptivo y correlacional. Es descriptivo por el hecho de describir cómo se manifiesta el problema de investigación, este a través de los datos e interpretación de las frecuencias y porcentajes. Asimismo, es de alcance correlacional porque tiene el objetivo de medir el nivel de relación entre la actitud emprendedora y la capacidad de creación de microempresas (Hernández et al., 2014)

\subsection{Diseño metodológico.}

El diseño metodológico está basado en un estudio no experimental de corte transversal. Los diseños no experimentales, no manipulan las variables de estudio, sino que persiguen encontrar las relaciones de las mismas, además es de corte transversal porque se realiza en un determinado momento (Hernández, et al., 2014). Bajo esta estrategia metodológica se plantea el estudio, la cual consta de sujetos con determinadas características (Ghozali, 2006; Niño, 2011), se contó con 131 estudiantes del VIII y IX ciclo de la carrera de administración de empresas de tres universidades de la Región de San Martin ubicadas al nororiente del Perú. Por las características de los sujetos, se contó con aquellos estudiantes que se encontraron en horario de clases, resumiéndose en un total de 131 encuestados según las universidades de estudio (ver tabla 1).

\section{Tabla 1. Muestra según universidad de estudio.}

\begin{tabular}{ccc}
\hline Universidades & Muestra & $\mathbf{\%}$ \\
\hline Universidad Científica del Perú (UCP) & 18 & $13.70 \%$ \\
\hline Universidad Alas Peruanas (UAP) & 51 & $38.90 \%$ \\
\hline Universidad Nacional de San Martín (UNSM) & 62 & $47.30 \%$ \\
\hline Total & $\mathbf{1 3 1}$ & $\mathbf{1 0 0 . 0 0 \%}$ \\
\hline
\end{tabular}

Fuente: elaboración propia (2021). 


\subsection{Instrumentos de recolección de los datos.}

Como instrumento de recolección de datos, se aplicó el cuestionario de actitud emprendedora y la capacidad de creación de microempresas. Se consideró el instrumento Entrepreneurial Attitude Orientation (EAO) Scale, desarrollado en Estados Unidos por Robinson (1987) y adaptado por Radovich (2017) que consta de 20 ítems, agrupa a la dimensión necesidad de logro (4 ítems), control sobre los hechos (4 ítems), creación e innovación (4 ítems), predisposición al riesgo (4 ítems) y la autoconfianza (4 ítems). En cuanto a la variable capacidad de creación de microempresa se partió del instrumento elaborado por (Veciana, 2005) y fue adaptado por (Radovich, 2017), consta de 21 ítems y está agrupado por la dimensión conocimiento empresarial ( 9 ítems), la experiencia empresarial (5 ítems) y la motivación empresarial (7 ítems).

Para la presente investigación se contó con la opinión de los expertos, quienes se encargaron de verificar en los instrumentos, la claridad, congruencia, contexto y dominio del constructo. Una vez que se contó con la verificación y aprobación, se procedió aplicar los instrumentos a las unidades de estudio. Como resultado se pudo constatar que los instrumentos cuentan con una fiabilidad de Alfa de Cronbach de 0.917 (capacidad emprendedora de microempresas) y 0.908 (actitud emprendedora), las que se consideran excelentes, concluyendo que los instrumentos son confiables, objetivos y aceptables para la investigación, está información se obtuvo haciendo uso de programa estadístico SPSS, el mismo que se utilizó para los análisis descriptivos y de correlación. 


\section{Resultados y discusión.}

Resultados descriptivos sociodemográficos.

En la tabla 2 se observa los resultados sociodemográficos, donde se puede ver que el género está representado por el $60.30 \%$ de mujeres y el 39.70\% de los hombres, respecto a la edad de los encuestados arrojaron que el 67.90\% están en las edades de 21 a 25 años, el $25.20 \%$ en edades de 16 a 20 años y solo el $6.90 \%$ tienen más de 25 años. Además, la tabla 2 revela los resultados según el lugar de procedencia, donde se destaca que el $83.20 \%$ es proveniente de la selva, el $9.90 \%$ proviene de la sierra y solo el $6.90 \%$ de la costa.

\section{Tabla 2. Datos sociodemográficos.}

\begin{tabular}{cccc}
\hline Variables & Categorías & Frecuencia & $\mathbf{\%}$ \\
\hline \multirow{3}{*}{ Género } & Masculino & 52 & $39.70 \%$ \\
\cline { 2 - 4 } & Femenino & 79 & $60.30 \%$ \\
\cline { 2 - 4 } & Total & 131 & $100 \%$ \\
\hline \multirow{3}{*}{ Edad } & $16-20$ años & 33 & $25.20 \%$ \\
\cline { 2 - 4 } & $21-25$ años & 89 & $67.90 \%$ \\
\cline { 2 - 4 } & 25 años a mas & 9 & $6.90 \%$ \\
\cline { 2 - 4 } & Total & 131 & $100.00 \%$ \\
\hline \multirow{2}{*}{$\begin{array}{c}\text { Lugar de } \\
\text { procedencia }\end{array}$} & Costa & 9 & $6.90 \%$ \\
\cline { 2 - 4 } & Sierra & 13 & $9.90 \%$ \\
\cline { 2 - 4 } & Selva & $\mathbf{1 3 1}$ & $\mathbf{1 0 0 \%}$ \\
\cline { 2 - 4 } & Total &
\end{tabular}

Fuente: elaboración propia (2021).

Análisis descriptivo de la variable actitud emprendedora y sus dimensiones.

En la tabla 3 se describe que a menudo existe una actitud emprendedora en los estudiantes de las tres universidades de la ciudad de Tarapoto. Se puede observar que la actitud 
emprendedora de manera general el $65.6 \%$ a menudo percibe tener una actitud emprendedora, el $26.7 \%$ siempre y solo el $7.6 \%$ algunas veces. Respecto a la necesidad del logro se podría decir que la percepción es positiva donde el $58.8 \%$ de los encuestados indicaron tener siempre una buena actitud frente a esta dimensión, el $35.9 \%$ reporto a menudo tienen necesidad del logro y solo el $5.3 \%$ menciono que algunas veces. En cambio, el 64.1\% señaló que a menudo tiene control sobre los hechos, a esto se sumó el $23.7 \%$ con siempre tiene control y el $11.5 \%$ algunas veces, sin embargo, el $0.8 \%$ nunca tiene control sobre los hechos.

La tabla 3 también revela los resultados de la dimensión creación e innovación, donde el 48.9\% siempre percibe tener creación de innovación, el $44.3 \%$ reportó que a menudo y solo el $6.9 \%$ menciono algunas veces. Asimismo, los resultados sobre la predisposición al riesgo, el 51.9\% menciono que a menudo está predispuesto, el $35.9 \%$ siempre y el $11.5 \%$ algunas veces, y solo el $0.8 \%$ indicó que nunca está predispuesto al riesgo. Finalmente, los resultados arrojaron que el $49.6 \%$ a menudo tiene autoconfianza, el $45.1 \%$ siempre la tiene, el $4.6 \%$ a menudo y solo el $0.8 \%$ reportó que nunca tiene autoconfianza. 
Tabla 3. Análisis descriptivo actitud emprendedora y sus dimensiones.

\begin{tabular}{|c|c|c|c|}
\hline Dimensiones & Categoría & Frecuencia & Porcentaje \\
\hline \multirow{4}{*}{ Actitud Emprendedora } & Algunas veces & 10 & $7.6 \%$ \\
\hline & A menudo & 86 & $65.6 \%$ \\
\hline & Siempre & 35 & $26.7 \%$ \\
\hline & Total & 131 & $100 \%$ \\
\hline \multirow{4}{*}{ Necesidad del logro } & Algunas veces & 7 & $5.3 \%$ \\
\hline & A menudo & 47 & $35.9 \%$ \\
\hline & Siempre & 77 & $58.8 \%$ \\
\hline & Total & 131 & $100 \%$ \\
\hline \multirow{5}{*}{ Control sobre los hechos } & Nunca & 1 & $0.8 \%$ \\
\hline & Algunas veces & 15 & $11.5 \%$ \\
\hline & A menudo & 84 & $64.1 \%$ \\
\hline & Siempre & 31 & $23.7 \%$ \\
\hline & Total & 131 & $100 \%$ \\
\hline \multirow{4}{*}{ Creación e innovación } & Algunas veces & 9 & $6.9 \%$ \\
\hline & A menudo & 58 & $44.3 \%$ \\
\hline & Siempre & 64 & $48.9 \%$ \\
\hline & Total & 131 & $100 \%$ \\
\hline \multirow{5}{*}{ Predisposición al riesgo } & Nunca & 1 & $0.8 \%$ \\
\hline & Algunas veces & 15 & $11.5 \%$ \\
\hline & A menudo & 68 & $51.9 \%$ \\
\hline & Siempre & 47 & $35.9 \%$ \\
\hline & Total & 131 & $100 \%$ \\
\hline \multirow{5}{*}{ Autoconfianza } & Nunca & 1 & $0.8 \%$ \\
\hline & Algunas veces & 6 & $4.6 \%$ \\
\hline & A menudo & 65 & $49.6 \%$ \\
\hline & Siempre & 59 & $45.1 \%$ \\
\hline & Total & 131 & $100 \%$ \\
\hline
\end{tabular}

Fuente: elaboración propia (2021). 
Microempresas y sus dimensiones.

En la tabla 4 se describe en general que a menudo (60.3\%), seguido de siempre $(35.9 \%)$ y algunas veces representado por el $2.8 \%$, indicaron que existe una capacidad de creación de microempresas en los estudiantes de las tres universidades en estudio, la cual también se indica la misma escala de valoración en sus dimensiones respectivamente.

Respecto a la dimensión conocimiento empresarial, del $100 \%$ de los encuestados el $60.3 \%$ reporto que a menudo tiene un conocimiento empresarial, seguido por siempre representado en un $26 \%$ y el $13.7 \%$ algunas veces suele tener conocimiento empresarial. Asimismo, los resultados respecto a la dimensión experiencia empresarial reportó que el $48.9 \%$ a menudo suele tener experiencia, seguido de la alternativa siempre por el $45.8 \%$ y algunas veces representado por el 4.6\%, sin embargo, el $0.8 \%$ reportó que nunca cuentan con una experiencia profesional en la capacidad de crear una empresa. Finalmente, la dimensión motivación empresarial mostró que el $55.7 \%$ se siente motivado con la capacidad de crear una microempresa, seguido del 36.6\% y solo el $7.6 \%$ algunas veces se mostró con una motivación empresarial. 


\section{Tabla 4. Análisis descriptivo capacidad de creación de} microempresas.

\begin{tabular}{cccc}
\hline Dimensiones & Escala & Frecuencia & Porcentaje \\
\hline \multirow{3}{*}{$\begin{array}{c}\text { Capacidad de creación de } \\
\text { microempresas }\end{array}$} & Algunas veces & 5 & $3.8 \%$ \\
\cline { 2 - 4 } & A menudo & 79 & $60.3 \%$ \\
\cline { 2 - 4 } & Siempre & 47 & $35.9 \%$ \\
\cline { 2 - 4 } Conocimiento empresarial & Total & $\mathbf{1 3 1}$ & $\mathbf{1 0 0 \%}$ \\
\cline { 2 - 4 } & Algunas veces & 18 & $13.7 \%$ \\
\cline { 2 - 4 } & A menudo & 79 & $60.3 \%$ \\
\cline { 2 - 4 } & Siempre & 34 & $26.0 \%$ \\
\hline \multirow{5}{*}{ Experiencia empresarial } & Total & $\mathbf{1 3 1}$ & $\mathbf{1 0 0 \%}$ \\
\cline { 2 - 4 } & Algunas veces & 1 & $0.8 \%$ \\
\cline { 2 - 4 } & A menudo & 64 & $4.6 \%$ \\
\cline { 2 - 4 } & Siempre & 60 & $48.9 \%$ \\
\cline { 2 - 4 } & Total & $\mathbf{1 3 1}$ & $\mathbf{1 0 0} \%$ \\
\hline \multirow{3}{*}{ Motivación empresarial } & Algunas veces & 10 & $7.6 \%$ \\
\cline { 2 - 4 } & A menudo & 73 & $55.7 \%$ \\
\cline { 2 - 4 } & Siempre & 48 & $36.6 \%$ \\
\cline { 2 - 4 } & Total & $\mathbf{1 3 1}$ & $\mathbf{1 0 0 \%}$ \\
\hline
\end{tabular}

Fuente: elaboración propia (2021).

Relación descriptiva del género, actitud emprendedora y capacidad de creación de microempresas.

En la tabla 5 se muestra un análisis descriptivo de la actitud emprendedora en relación con el género de estudio, donde se aprecia que el 38,2\% del género femenino muestra una mejor actitud emprendedora, a diferencia del género masculino que solo mostro una mejor actitud emprendedora en un $25.2 \%$. en general la actitud emprendedora y el género se muestra que $63.4 \%$ de los hombre y mujeres tienen una mejor actitud emprendedora, 
seguido del $35.9 \%$ que mostró una actitud regular y solo el $0.8 \%$ se mostró con una mala actitud.

\section{Tabla 5. Análisis entre la actitud emprendedora y género de estudio.}

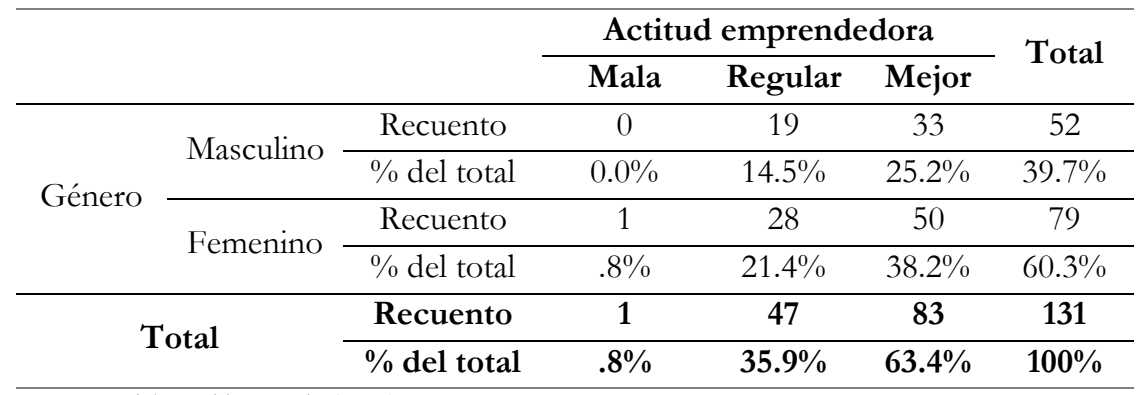

Fuente: elaboración propia (2021).

Asimismo, la tabla 6 muestra la relación de la capacidad de creación de microempresas y género, donde se observa que el $38.2 \%$ del género femenino se mostró con una mejor actitud frente a la capacidad de creación de microempresas, en cambio el 24.4\% del género masculino mostró una actitud mejor en la capacidad de creación de una micro y pequeña empresa. La relación de manera general arrojo que el 62.6\% de los encuestados de ambos géneros mostraron tener una mejor actitud en la creación de microempresas, el 36.6\% se mostró con una actitud regular y solo el $0.8 \%$ mostró una mala actitud en la capacidad para crear una microempresa. 


\section{Tabla 6. Análisis entre la capacidad de creación de microempresas y género de estudio.}

\begin{tabular}{|c|c|c|c|c|c|c|}
\hline & & & \multicolumn{3}{|c|}{$\begin{array}{l}\text { Capacidad de creación } \\
\text { de microempresas }\end{array}$} & \multirow[t]{2}{*}{ Total } \\
\hline & & & Mala & Regular & Mejor & \\
\hline \multirow{4}{*}{ Género } & \multirow{2}{*}{ Masculino } & Recuento & 1 & 19 & 32 & 52 \\
\hline & & $\%$ del total & $.8 \%$ & $14.5 \%$ & $24.4 \%$ & $39.7 \%$ \\
\hline & \multirow{2}{*}{ Femenino } & Recuento & 0 & 29 & 50 & 79 \\
\hline & & $\%$ del total & $0.0 \%$ & $22.1 \%$ & $38.2 \%$ & $60.3 \%$ \\
\hline \multirow{2}{*}{\multicolumn{2}{|c|}{ Total }} & Recuento & 1 & 48 & 82 & 131 \\
\hline & & $\%$ del total & $.8 \%$ & $36.6 \%$ & $62.6 \%$ & $100 \%$ \\
\hline
\end{tabular}

Fuente: elaboración propia (2021).

Relación descriptiva de la edad, actitud emprendedora y capacidad de creación de microempresas.

En la tabla 7 se muestra un análisis descriptivo de la actitud emprendedora y el rango de edades de los estudiantes universitarios, donde se observa que el $43.5 \%$ de las edades entre 21 y 25 tienen una mejor actitud emprendedora, seguido del 14.5\% de entre 16 a 20 años y solo el 5.3\% de 25 años a más mostró tener una mejor actitud emprendedora, es decir, en general el $63,4 \%$ de los estudiantes universitarios mostraron tener una mejor actitud emprendedora, seguido del 35.9\% que mostró una actitud regular y solo el $0.8 \%$ mostro una actitud mala. 
Tabla 7. Análisis entre la actitud emprendedora y edad.

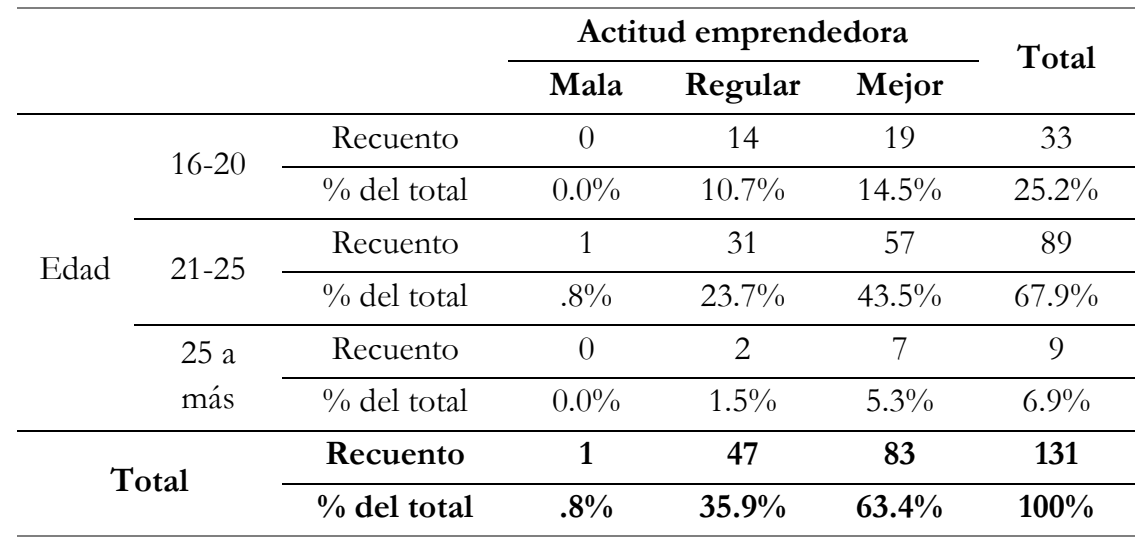

Fuente: elaboración propia (2021).

La tabla 8 muestra los resultados de capacidad de creación de microempresas en relación con las edades de los estudiantes, donde se observa que $44.3 \%$ de 21 y 25 años mencionaron tener una mejor capacidad de creación de microempresas, seguido por el $13 \%$ de entre 16 y 20 años y solo el 5.3\% de más de 25 años mostraron también tener una mejor capacidad para crear microempresas. En general, el $62.6 \%$ señalan tener una mejor capacidad de creación de microempresas, el 36.6\% indican tener una regular capacidad y solo el $0.8 \%$ indican tener mala capacidad para crear una microempresa. 


\section{Tabla 8. Análisis entre la capacidad de creación de microempresas y la edad del estudio.}

\begin{tabular}{|c|c|c|c|c|c|c|}
\hline & & & \multicolumn{3}{|c|}{$\begin{array}{l}\text { Capacidad de creación de } \\
\text { microempresas }\end{array}$} & \multirow[t]{2}{*}{ Total } \\
\hline & & & Mala & Regula & Mejor & \\
\hline \multirow{6}{*}{ Edad } & \multirow{2}{*}{$16-20$} & Recuento & 0 & 16 & 17 & 33 \\
\hline & & $\%$ del total & $0.0 \%$ & $12.2 \%$ & $13.0 \%$ & $25.2 \%$ \\
\hline & \multirow{2}{*}{$21-25$} & Recuento & 1 & 30 & 58 & 89 \\
\hline & & $\%$ del total & $.8 \%$ & $22.9 \%$ & $44.3 \%$ & $67.9 \%$ \\
\hline & \multirow{2}{*}{$\begin{array}{l}25 \mathrm{a} \\
\mathrm{mas}\end{array}$} & Recuento & 0 & 2 & 7 & 9 \\
\hline & & $\%$ del total & $0.0 \%$ & $1.5 \%$ & $5.3 \%$ & $6.9 \%$ \\
\hline \multirow{2}{*}{\multicolumn{2}{|c|}{ Total }} & Recuento & 1 & 48 & 82 & 131 \\
\hline & & $\%$ del total & $.8 \%$ & $36.6 \%$ & $62.6 \%$ & $100 \%$ \\
\hline
\end{tabular}

Fuente: elaboración propia (2021).

Relación entre las dimensiones de capacidad de creación de microempresas y la actitud emprendedora.

Previo a la prueba de hipótesis de correlación, los datos pasaron por un proceso de revisión de los supuestos para realizar este tipo de estudios de correlación, entre ellos la prueba de normalidad a través de Kolmogorov Smirnov (K-S). Se identificó que los valores $\mathrm{K}-\mathrm{S}$ de las variables y dimensiones son menores a 0.05 ( $\mathrm{p}<0.05)$, por lo tanto, no cumplen con una distribución normal, tomando la decisión de considerar un análisis estadístico no paramétrico a través del coeficiente de correlación Rho de Spearman.

La tabla 9 muestra la correlación de la actitud emprendedora y capacidad de creación de microempresas, cuyo coeficiente Rho Spearman es igual a 0.656 con un p-valor igual a 0.000 (p-valor $<0.05$ ) indicando una correlación positiva moderada, lo que significa, que a mejor actitud emprendedora 
mayor creación de microempresas en los estudiantes de las universidades (UCP, UAP Y UNSM).

Con respecto a la correlación actitud emprendedora y conocimiento empresarial se obtuvo un coeficiente Rho Spearman igual a 0.565 y un p valor igual a 0.000 (p-valor $<0.05)$ correlación positiva moderada, es decir, que a mejor actitud emprendedora mayores conocimientos empresariales habrá en los estudiantes universitarios (UCP, UAP y UNSM). Asimismo, la correlación entre actitud emprendedora y experiencia empresarial se obtuvo un nivel de correlación y un grado de significancia positiva moderada (Rho $=0.552 * * ; \mathrm{p}=0.000)$.

Es decir, a mejor grado de actitud emprendedora mayor será la experiencia empresarial en los estudiantes universitarios (UCP, UAP y UNSM). Finalmente, la correlación actitud emprendedora y motivación empresarial se obtuvo una correlación positiva media alta con un nivel de significancia positivo $y$ significativo $(\mathrm{Rho}=0.686 * * ; \mathrm{p}=0.000)$, lo que significa que la actitud emprendedora y la motivación empresarial tienen una correlación positiva media alta y significativa, es decir, que a mejor grado de actitud emprendedora mayor será la motivación empresarial en los estudiantes de las tres universidades. 


\section{Tabla 9. Análisis de correlaciones.}

\begin{tabular}{|c|c|c|c|}
\hline \multirow[t]{2}{*}{ Variables y dimensiones } & \multicolumn{3}{|c|}{ Actitud emprendedora } \\
\hline & Rho & p-valor & $\mathbf{N}$ \\
\hline $\begin{array}{l}\text { Capacidad de creación } \\
\text { microempresas }\end{array}$ & $0.656^{* *}$ & 0.000 & 131 \\
\hline Conocimiento empresarial & $0.565^{* *}$ & 0.000 & 131 \\
\hline Experiencia empresarial & $0.552^{* *}$ & 0.000 & 131 \\
\hline Motivación empresarial & $0.686^{* *}$ & 0.000 & 131 \\
\hline
\end{tabular}

**. La correlación es significativa en el nivel 0.01 (bilateral).

Fuente: elaboración propia (2021).

El principal objetivo del presente estudio fue determinar la relación que existe entre la actitud emprendedora y la capacidad de creación de microempresas en estudiantes de la carrera de administración tres universidades (UCP, UAP y UNSM), ubicadas en la ciudade Tarapoto, de la región San Martin ubicadas al nororiente peruano.

$\mathrm{Al}$ analizar los resultados, se encontró mediante el análisis estadístico Rho de Spearman un p valor $<0.000$ ( $\mathrm{p}$ - valor $<0.05$ ) con un coeficiente de correlación de $0.656^{* *}$, por lo tanto se menciona que si existe relación entre las variables de estudio, lo cual indica que a mejor actitud emprendedora mayor es la capacidad de creación de microempresas en los estudiantes. Se puede contrastar con los resultados encontrados en el estudio de Radovich (2017), donde la actitud emprendedora y la capacidad para crear microempresas tienen una alta relación. Asimismo, Mitma (2018), señala que el espiritu emprendedor y la reación de micro y pequeñas empresas tienen una relación positiva alta. Por lo tanto, la actitud emprendedora de los estudiantes es determinante para realizar emprendimientos.

En cuanto a la dimensión conocimiento empresarial, se encontró que se relaciona de manera moderada y significativa con 
la actitud emprendedora (Rho $=0.565 * * ; p<0.000)$ de los cuales cuanto mayor sea la actitud emprendedora mayor experiencia empresarial tendrán los estudiantes. Estos resultados se pueden contrastar con el estudio de Loli et al. (2014) donde se constato que el conocimiento empresarial y la inntención emprendedora tienen una relación positiva y significativa. A esto se suma Radovich (2017) donde revela que un $77.7 \%$ de los encuestados tienen un nivel óptimo de conocimientos para tomar decisiones, negociar, comunicar, liderar y motivar su microempresa. Por lo que se infiere que el conocimiento empresarial es un factor determinante para que los estudiantes universitarios tomen la decición de emprender.

La experiencia empresarial dentro de un emprendimiento empresarial tambien es un facctor importante, los resultados revelan una relaciona moderada y significativa con la actitud emprendedora $(\mathrm{Rho}=0.552 * * ; \mathrm{p}<0.000)$, esto significa, cuanto mayor sea la actitud emprendedora mayor serán los conocimientos empresariales adquiridos, por ende serán capaces de emprender. Esto corrobora a los resultados de Loli, Del Carpio, y Vergara (2014) donde mencionan que la experiencia laboral está asociada al nivel de emprendimiento. Asimismo, Radovich (2017), encontró $78.3 \%$ de los estudantes cuentan con un nivel optimo de experiencia empresarial para tomar mejores decisiones, mejorar ideas, conocer el mercado laboral y aplicarlo en una microempresa.

Los resultados mostraron que la motivación empresarial está relacionada de manera positiva moderada y significativa con la actitud emprendedora (Rho $=0.686 * ; \mathrm{p}<0.000)$, lo que se infiere que a mayor actitud emprendedora existirá mayor motivación laboral. Al respecto Peralta (2018) menciona que no solo existe una relación entre estos constructos sino que la 
motivación es una componente de influencia para la capacidad de emprender. Por consiguinete Radovich (2017) encontro que el $83.7 \%$ de los estudiantes están en un nivel óptimo de motivación laboral para tener una visión de negocios, contribuir con la sociedad, solucionar sus propios problemas económicos y superar las barreras que se le presenten en su microempresa.

\section{Conclusiones.}

Los resultados del presente estudio permiten concluir que la presente investigación hace una contribucion en la confirmación de que la actitud emprendedora y la capacidad de creación de microempresas tienen una relación positiva moderada y significativa en los estudiante de administración de empresas de las tres universidades, por lo tando, los resultados empiricos del presente estudio aportan al conocimiento del area empresarial y organizacional, donde se determina que la actitud emprendedora es relevante para la cración de microempresas por los estudiantes universitarios. Sin embargo, se requiere medir otras dimensiones que intervienen en la capaccidad de creación de las empresas, como el entorno y su relación con los procesos de creación de esta.

Los resultado tambien mostraron que existe correlación entre la actitud emprendedora y las dimensiones de la capacidade de creación de microempresas, por lo que se infiere que las universidades deben seguir manteniendo y mejorando las asignaturas de emprendedorismo para que los estudiantes sean capaces de arriesgar y crear microempresas. Además, es necesario que las universidades (UCP, UAP Y UNSM) tengan en cuenta la actitud emprendedora de sus estudiantes y establescan estrategias para poner en práctica lo aprendido, ademas adquieran nuevas experiencias empresariales, determinandose por lo general que a 
mejor actitud emprendedora mayor capacidad de creación de microempresas. Partiendo de esta premisa, es necesario que las universidades verifiquen los planes curriculares establecidos y adpten al cotexto de emprendimientos de la region, ademas de vigilar la actitud emprendedora para que se sientan motivados y logren el éxito empresarial.

\section{Referencias bibliográficas.}

Al-Mamun, A.; Fazal, S. y Muniady, R. (2019). Entrepreneurial knowledge, skills, competencies and performance. Asia Pacific Journal of Innovation and Entrepreneurship, 13(1), 29-48. https://doi.org/10.1108/APJIE-11-2018-0067

Al-Mamun, A.; Subramaniam, E.; Che-Nawi, N. y Binti-Zainol, N. (2016). Entrepreneurial Competencies and Performance of Informal Micro-Enterprises in Malaysia. Mediterranean Journal Of Social Sciences, 7(3), 273-281.

Ato, M.; López-García, J. y Benavente, A. (2013). Un sistema de clasificación de los diseños de investigación en psicología. Anales de Psicología, 29(3), 1038-1059. https://doi.org/10.6018/analesps.29.3.178511

Contreras, A. y Macías, P. (2021). Percepción de los estudiantes sobre la intención de emprender. Caso: Universidad de Guadalajara, México. SUMMA. Revista disciplinaria en Ciencias económicas y sociales, 3(1), 1-25. https://doi.org/10.47666/summa.3.1.03

Durán-Aponte, E. y Arias-Gómez, D. (2016). Actitud emprendedora y estilos emocionales. Contribuciones para el diseño de la formación de futuros emprendedores. Gestión de La Educación, $6(2)$, 83. https://doi.org/10.15517/rge.v1i2.25490 
El Comercio (2018). Perú es el quinto país más emprendedor en el mundo. Recuperado de: https://elcomercio.pe/economia/peru/peru-quinto-paisemprendedor-mundo-noticia-532878-noticia/

Gestión (2018). Perú es el país con mayor espíritu emprendedor en América Latina y el quinto en el mundo. Recuperado de: https://gestion.pe/economia/peru-pais-mayor-espirituemprendedor-america-latina-quinto-mundo-237414-noticia/

Ghozali, I. (2006). Structural equation modeling. Metode alternative dengan partial least squares. Semarang: Diponegoro University Publishing Agency.

Hernández, R.; Fernández, C. y Baptista, M. (2014). Metodología de la investigación. México, D.F.: Editorial McGraw-Hill Education.

INEI (2018). Perú: Indicadores de Educación por departamento, 2007-2017. Lima: Instituto Nacional de Estadística e Informática.

INEI (2019). En el Perú existen cerca de dos millones y medio de empresas a diciembre de 2018. Recuperado de: http://m.inei.gob.pe/media/MenuRecursivo/noticias/notade-prensa-n-034-2019-demografia-empresarial.pdf

Loli, A.; Dextre, E.; Del Carpio, J. y La Jara, E. (2014). Actitudes de creatividad y emprendimiento en estudiantes de la Universidad Nacional de Ingeniería y su relación con algunas variables socio demográficas. Revista de Investigación En Psicología, 13(2), 139. https://doi.org/10.15381/rinvp.v13i2.3722

Loli, A.; Del Carpio, J. y Vergara, A. (2014). Actitudes de emprendimiento, necesidad de logro y la intención de desarrollar un negocio en estudiantes de universidades públicas de Lima Metropolitana. Revista de Investigación En 
Psicología, 15(1), 61.

https://doi.org/10.15381/rinvp.v15i1.3662

Martínez, J.; Durán, S. y Serna, W. (2021). COVID-19, educación en emprendimiento e intenciones de emprender: Factores decisorios en estudiantes universitarios. Revista de ciencias sociales, $27(2)$, 272-283. https://doi.org/10.31876/rcs.v27i2.35913

Mitma, R. (2018). Espíritu emprendedor y la creación de micro y pequeñas empresas en las estudiantes de la carrera profesional de educación inicial intercultural - Instituto de Educación Superior Pedagógico Publico - Huancavelica. Trabajo de grado. Universidad Nacional de Huancavelica, Perú.

Muñóz, Á. y Martínez, L. (2020). Emprendimiento social y felicidad urbana. SUMMA. Revista disciplinaria en Ciencias económicas y sociales, 2(1), 127-169.

Nabiswa, F. y Mukwa, J. (2017). Impact of credit financing on human resource development among micro and small enterprises: a case study of Kimilili Sub County, Kenya. Asian Journal OfManagement Science and Economics, 4(1), 43-53.

Niño, V. (2011). Metologia de la Investigación: Diseño y ejecución. Bogotá: Ediciones de la U.

Olozagaste, J.; Calderón, Y. y Espinosa, M. (2017). Formación del Espíritu Empresarial en Jóvenes Mexicanos: Retos y Oportunidades en la Educación Media Superior. Revista Latinoamericana de Educación Inclusiva, 11(2), 163-177. https://doi.org/10.4067/S0718-73782017000200011

Ortiz, P. (2016). Actitud emprendedora de los jóvenes españoles en un contexto de crisis. Revista de Ciencias Sociales, 22(1), 139-152. https://doi.org/10.31876/rcs.v22i1.24902 
Peralta, L. (2018). La influencia de la motivación en las capacidades emprendedoras de las estudiantes del primer año de secundaria. Trabaj de grado. Universidad César Vallejo, Perú.

Radovich, J. (2017). La actitud emprendedora y su relación con la capacidad de creación de microempresas en los estudiantes de administración de la Universidad Inca Garcilaso de la Vega. Trabajo de grado. Universidad Nacional de Educación Enrique Guzmán y Valle, Perú.

Robinson, P. (1987). Prediction of entrepreneurship based on an attitude consistency model. Utah: University of Brigham Young.

Serida, J.; Guerrero, C.; Alzamora, J.; Borda, A. y Morales, O. (2018). Global Entrepreneurship Monitor: Perú 2017-2018. Perú: ESAN Ediciones.

Texto único ordenado de la ley de impulso al desarrollo productivo y al crecimiento empresarial, Pub. L. No. 0132013-PRODUCE, 1 (2013). Recuperado de: http://www2.produce.gob.pe/dispositivos/publicaciones/d s013-2013-produce.pdf

Veciana, J. (2005). La creación de empresas. Un enfoque gerencial. Barcelona: In la Caixa.

Zea-Fernández, R.; Benjumea-Arias, M. y Valencia-Arias, A. (2020). Metodología para la identificación de las capacidades dinámicas para el emprendimiento en Instituciones de Educación Superior. Ingeniare. Revista Chilena de Ingeniería, 28(1), 106-119. https://doi.org/10.4067/S071833052020000100106 